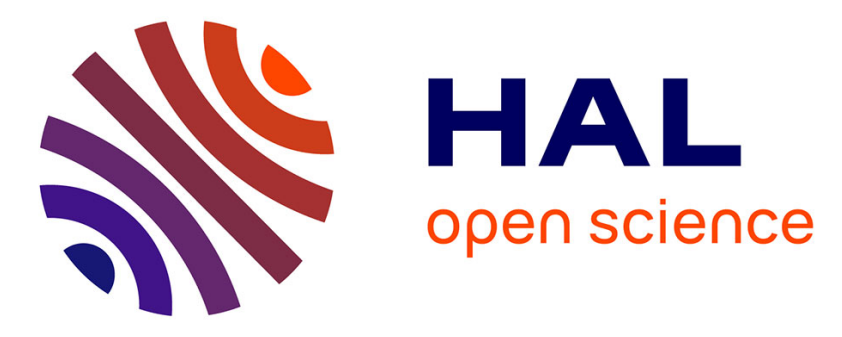

\title{
Nonlinear state-feedback with disturbances estimation for DC-DC Buck converter
}

\author{
Mamadou Lamine Fall, Olivier Gehan, Eric Pigeon, Mathieu Pouliquen, \\ Mohammed M'Saad
}

\section{- To cite this version:}

Mamadou Lamine Fall, Olivier Gehan, Eric Pigeon, Mathieu Pouliquen, Mohammed M'Saad. Nonlinear state-feedback with disturbances estimation for DC-DC Buck converter. 2nd International Symposium on Environment-Friendly Energies and Applications, Jun 2012, United Kingdom. hal01057099

\section{HAL Id: hal-01057099 \\ https://hal.science/hal-01057099}

Submitted on 21 Aug 2014

HAL is a multi-disciplinary open access archive for the deposit and dissemination of scientific research documents, whether they are published or not. The documents may come from teaching and research institutions in France or abroad, or from public or private research centers.
L'archive ouverte pluridisciplinaire HAL, est destinée au dépôt et à la diffusion de documents scientifiques de niveau recherche, publiés ou non, émanant des établissements d'enseignement et de recherche français ou étrangers, des laboratoires publics ou privés. 


\title{
Nonlinear state-feedback with disturbances estimation for DC-DC Buck Converter
}

\author{
L. Fall, O. Gehan, E. Pigeon, M. Pouliquen, M. M’Saad
}

\begin{abstract}
The aim of this paper is to present new results on the Buck converter control. The behaviour of the buck converter is described by the well-known average model and the control strategy is based on a double integral high gain control law including an estimation of current load. This control scheme rejects efficiently the disturbances introduced by the battery dynamics that depends on both the coil current and its charge level. Of particular interest, this nonlinear control algorithm ensures on the concerned open subset exponentially convergence of the state errors on the entire domain in a free disturbance case. The control law performances are evaluated regarding load and supply variations.
\end{abstract}

\section{INTRODUCTION}

Cont ontrol of DC / DC buck converters has been highly investigated in the two last decades for two main reasons. Firstly, these components are actually used in many practical applications of great interest especially electrical vehicles and embedded systems. In a second way, these converters have nonlinear dynamics and a discontinuous behaviour which is of great investigation interest in the field of nonlinear control. Its rapid structure variation is accounted for using averaged models [1], [2]. Based on the latest one, different nonlinear control techniques have been developed, including passivity techniques [2], sliding mode [3], backstepping [4], feedback linearization and, more generally, flatness methods [5].

All these approaches have the same objective that is to say controlling the converter output voltage despite the variations of the different physical variables governing its behavior. The control scheme performance is mainly studied as regards the uncertainties on the components and the load current disturbances and the changes in supply voltage. In fact, the latest one is inherent to embedded systems because the energy storage device is usually a battery that provides a voltage which depends on the supplied current and its charge level.

In general, the proposed algorithms attenuate the effects of disturbances thanks to their intrinsic properties or by

Authors are with the Groupe de Recherche en Informatique, Image, Automatique et Instrumentation de Caen (GREYC), ENSICAEN and Caen University, 14050 Caen, FRANCE (corresponding author to provide phone: 33-231452707; e-mail: olivier.gehan@ensicaen.fr). adapting the control law with a measure of the considered disturbance.

In this paper, the main objective is the control of the converter output voltage in the presence of two types of disturbances. The first one, namely the input voltage variations induced by the charge level, may be considered in a first approximation as ramp-like type and can consequently be taken into account with a double integral action. The second ones are the load current variations and they can be rejected using a feed-forward action based on an online estimation of the load current and its first derivative [6]. In fact, the average model of the buck converter exhibits a minimum-phase behavior which allows such an approach.

This paper is organized as follows. In the second section, the average buck converter model is presented and its nonlinear state-space representation is given. A nonlinear high gain observer is synthesized to deliver on line estimation of the unmeasured variables. In Section 3 a change of variables is introduced to conduct the original model into the control model which is used to synthesize the proposed high gain control law. A feed-forward type action is introduced to perform well in the presence of specific disturbances. Section 4 is devoted to the simulation results which are discussed for both the observer and the control law performances. Conclusions are given in the last section.

\section{BUCK CONVERTER: MODELING AND OBSERVER DESIGN}

\section{A. Buck Modeling}

The Buck DC / DC converter which is considered in this paper is described on figure 1 . The average electrical scheme includes a coil $(L)$ with its internal resistor $\left(\mathrm{R}_{\mathrm{L}}\right)$, a diode, a capacitor $(C)$ and a controlled power switch. The average buck converter model can be described by the following dynamical system

$$
\left\{\begin{array}{l}
\dot{v}_{c}(t)=\frac{1}{C}\left(i_{L}(t)-i(t)\right) \\
\dot{i}_{L}(t)=-\frac{1}{L}\left(R_{L} i_{L}(t)+v_{c}(t)-u(t) v_{e}(t)\right)
\end{array}\right.
$$

where $i_{L}(t)$ and $v_{c}(t)$ respectively denote the input current and the output voltage, $i(t)$ denotes the load current. The control variable is $u(t)$ and is related to the switch duty cycle. Finally, $v_{e}(t)$ is the input DC/DC voltage and belongs to the 
following interval $v_{e}(t) \in\left[\begin{array}{ll}v_{e \min } & v_{e \max }\end{array}\right]$ with $v_{e \max }=v_{e}^{*}$ its nominal value.

The control objective consists in keeping the output voltage $v_{c}(t)$ in the neighborhood of a pre-specified constant reference value $v_{c}^{*}$ independently of the different disturbances that are step-like ones and they correspond to the variations of the current $i(t)$ as well as the input voltage $v_{e}(t)$.

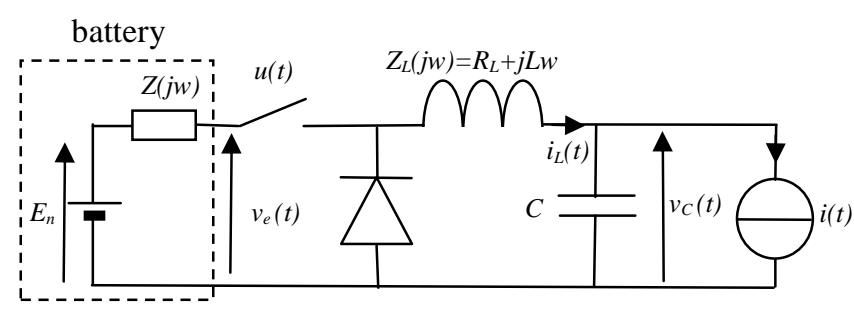

Fig. 1. Schematic Buck converter.

The control model can be described by the following state space model

$$
\begin{aligned}
& \left\{\begin{array}{l}
\dot{x}(t)=F x(t)+G u(t)+M i(t) \\
v_{c}(t)=H X(t)
\end{array}\right. \\
& \text { with } x(t)=\left(\begin{array}{l}
x_{1}(t) \\
x_{2}(t)
\end{array}\right)=\left(\begin{array}{l}
v_{c}(t) \\
i_{L}(t)
\end{array}\right) \text { and } F \text { and } G \text { are defined as }
\end{aligned}
$$

follows

$$
\begin{aligned}
& F=\left(\begin{array}{cc}
0 & 1 / C \\
-1 /\left(R_{L} L\right) & -1 / L
\end{array}\right), G=\left(\begin{array}{c}
0 \\
v_{e}(t) / L
\end{array}\right) \\
& \mathrm{M}=\left(\begin{array}{c}
-1 / C \\
0
\end{array}\right), H=\left(\begin{array}{ll}
1 & 0
\end{array}\right)
\end{aligned}
$$

The varying-time equilibrium point of (1) associated to $v_{c}^{*}$ is given by

$$
\left\{\begin{array}{l}
v_{c}(t)=v_{c}^{*}=x_{1}^{*} \\
i_{L}(t)=i_{L}^{*}(t)=x_{2}^{*}(t)=i(t) \\
u^{*}(t)=\frac{L}{v_{e}^{*}}\left(\frac{d i(t)}{d t}+\frac{R_{L}}{L} i(t)+\frac{v_{c}^{*}}{L}\right)
\end{array}\right.
$$

\section{B. Observer design}

As we shall see later, the expression of the proposed control law includes the variable $u^{*}(t)$ which depends on both the load current and its first derivative. One of the objectives is to design a load current sensor-less nonlinear control law for the on line monitoring of the output voltage of the buck DC / DC converter. Consequently, one shall use an observer which provides on-line estimate of both $i(t)$ and its derivative. Such an observer can be designed using the sole output voltage and coil current measurements.

For that purpose, let us first define the following state space vector

$$
x_{o}(t)=\left(\begin{array}{c}
x_{o 1}(t) \\
x_{o 2}(t) \\
x_{o 3}(t)
\end{array}\right)=\left(\begin{array}{c}
x_{1}(t) \\
-\frac{i(t)}{C} \\
-\frac{1}{C} \frac{d i(t)}{d t}
\end{array}\right)
$$

Using the output voltage dynamics (1), the observer design model can then be written under the following canonical and uniformly observable form

$$
\left\{\begin{array}{l}
\dot{x}_{o 1}(t)=\frac{1}{C} i_{L}(t)+x_{o 2}(t) \\
\dot{x}_{o 2}(t)=x_{o 3}(t) \\
\dot{x}_{o 3}(t)=\eta
\end{array}\right.
$$

where $\eta$ denotes an unknown constant whose upper bound is denoted by $\delta$. The underlying load current model is motivated by the class of disturbances the control scheme as to deal with, namely ramp-like variations. System (6) belongs to the class of systems proposed in [8] and for which the underlying high gain observer has been proposed

$$
\left\{\begin{array}{l}
\dot{\hat{x}}_{o 1}(t)=\frac{1}{C} i_{L}(t)+\hat{x}_{o 2}(t)-3 \theta\left(\hat{x}_{o 1}(t)-x_{o 1}(t)\right) \\
\dot{\hat{x}}_{o 2}(t)=\hat{x}_{o 3}(t)-3 \theta^{2}\left(\hat{x}_{o 1}(t)-x_{o 1}(t)\right) \\
\dot{\hat{x}}_{o 3}(t)=-\theta^{3}\left(\hat{x}_{o 1}(t)-x_{o 1}(t)\right)
\end{array}\right.
$$

where $\hat{x}_{o i}(t)$ denotes the estimation of $x_{o i}(t)$ with $i \in\{1,2,3\}$ and $\theta$ is the observer design parameter. Using observer (7), the estimation $\hat{u}^{*}(t)$ of $u^{*}(t)$ can be easily obtained

$$
\hat{u}^{*}(t)=\frac{L}{v_{e}^{*}}\left(-C \hat{x}_{o 3}(t)-C \frac{R_{L}}{L} \hat{x}_{o 2}(t)+\frac{v_{c}^{*}}{L}\right)
$$

Under the hypothesis that $\delta$ is bounded, the trajectories of observer (7) satisfy the following property [11] :

$$
\begin{aligned}
& \exists \theta_{0}>0 ; \forall \theta \geq \theta_{0} ; \exists \mu_{0}(\theta), \eta_{0}(\theta)>0 \text { such that } \forall t \geq 0: \\
& \left\|\hat{x}_{0}(t)-x_{0}(t)\right\| \leq \eta_{0}(\theta) e^{-\mu_{0}(\theta) t}\left\|\hat{x}_{0}(0)-x_{0}(0)\right\|+m \frac{\delta}{\theta}
\end{aligned}
$$

where $m>0$ is real constant. In this case, the estimation error remains in a ball whose radius can be made as small as desired by choosing $\theta$ high enough. This implies that the error of estimation $\tilde{u}^{*}(t)=\hat{u}^{*}(t)-u^{*}(t)$ also remains in a ball whose radius depends on $\theta$. In fact, one has

$$
\left|\hat{x}_{0 i}(t)-x_{0 i}(t)\right| \leq\left\|\hat{x}_{0}(t)-x_{0}(t)\right\| \forall i \in\{1,2,3\}
$$

Hence, using (8), (9) and (10), one obtains

$$
\left|\tilde{u}^{*}(t)\right| \leq \frac{L C}{v_{e}^{*}}\left(1+\frac{R_{L}}{L}\right)\left(\eta_{0}(\theta) e^{-\mu_{0}(\theta) t}\left\|\hat{x}_{0}(0)-x_{0}(0)\right\|+m \frac{\delta}{\theta}\right)
$$




\section{CONTROL ALGORITHM}

\section{A. Control Model}

To design the controller, let us define the following change of variables

$\psi: \mathfrak{R}^{+} \times \mathfrak{R}^{+} \stackrel{\psi}{\rightarrow} \mathfrak{R}^{+} \times \mathfrak{R}^{+}, x(t) \stackrel{\psi}{\rightarrow} w(t)=\left(\begin{array}{l}w_{1}(t) \\ w_{2}(t)\end{array}\right)=\Lambda x(t)$

where

$\Lambda=\left(\begin{array}{ll}C & 0 \\ 0 & 1\end{array}\right)$

The control objective can be expressed in the new coordinates where the variables $w_{1}(t)$ and $w_{2}(t)$ should be kept in the neighborhood of a pre-specified couple of reference values defined as

$$
\left\{\begin{array}{l}
w_{1}^{*}=C x_{1}^{*} \\
w_{2}^{*}(t)=x_{2}^{*}(t)
\end{array}\right.
$$

The underlying control variable $u(t)$ must be synthesized to ensure that

$$
\lim _{t \rightarrow \infty} \tilde{w}_{i}(t)=w_{i}(t)-w_{i}^{*}=0 \quad \forall \mathrm{i} \in\{1,2\}
$$

According to (2) and (9), the dynamics of the tracking errors can be expressed as

$$
\left\{\begin{aligned}
\dot{\tilde{w}}_{1}(t) & =\dot{w}_{1}(t)=C \dot{x}_{1}(t)=x_{2}(t)-x_{2}^{*}(t)=\tilde{w}_{2}(t) \\
\dot{\tilde{w}}_{2}(t) & =\frac{v_{e}(t)}{L}\left(u(t)-u^{*}(t)\right) \\
& -\frac{R_{L}}{L} \tilde{w}_{2}(t)-\frac{1}{L C} \tilde{w}_{1}(t)
\end{aligned}\right.
$$

In order to compensate the ramp-like disturbances, the control algorithm must integrate a double integral action. Consequently, and using (14), the control model is given by

$$
\left\{\begin{array}{l}
\dot{\gamma}(t)=\sigma(t) \\
\dot{\sigma}(t)=\tilde{w}_{1}(t) \\
\dot{\tilde{w}}_{1}(t)=\tilde{w}_{2}(t) \\
\dot{\tilde{w}}_{2}(t)=\frac{v_{e}(t)}{L}(u(t)-u *(t))-\frac{R_{L}}{L} \tilde{w}_{2}(t)-\frac{1}{L C} \tilde{w}_{1}(t)
\end{array}\right.
$$

\section{B. Observer-Based High Gain Control Law}

The control model (15) can be written under the following canonical form

$$
\dot{z}(t)=A z(t)+B \frac{v_{e}(t)}{L}\left(u(t)-u^{*}(t)\right)+\varphi(z(t))
$$

with

$$
z(t)=\left(\begin{array}{c}
z_{1}(t) \\
z_{2}(t) \\
z_{3}(t) \\
z_{4}(t)
\end{array}\right)=\left(\begin{array}{c}
\gamma(t) \\
\sigma(t) \\
\tilde{w}_{1}(t) \\
\tilde{w}_{2}(t)
\end{array}\right)
$$

$$
A=\left(\begin{array}{cc}
0_{3 \times 1} & I_{3 \times 3} \\
0_{1 \times 1} & 0_{1 \times 3}
\end{array}\right), B=\left(\begin{array}{c}
0_{3 \times 1} \\
1
\end{array}\right)
$$

$$
\varphi(z(t))=\left(\begin{array}{c}
0 \\
0 \\
0 \\
-\frac{1}{L C} z_{3}(t)-\frac{R_{L}}{L} z_{4}(t)
\end{array}\right)
$$

The state-feedback high gain control law based on the control model (16) and using the estimation of the current load and its derivative as well as the input voltage measurement can be written as follows

$$
u(t)=\hat{u}^{*}(t)+\frac{L}{v_{e}^{*}} v(z(t))
$$

with

$$
v(z(t))=-k_{c} B^{T} \bar{S} \Gamma_{\lambda} z(t)
$$

where $\Gamma_{\lambda}$ is the following diagonal matrix

$$
\Gamma_{\lambda}=\operatorname{diag}\left(\lambda^{4}, \lambda^{3}, \lambda^{2}, \lambda\right)
$$

with $\lambda \in \mathfrak{R}^{+}$and $\bar{S}$, the unique symmetric positive definite solution of the following algebraic Lyapunov equation

$$
\begin{aligned}
& \bar{S}+A^{T} \bar{S}+\bar{S} A=\bar{S} B B^{T} \bar{S} \\
& \text { and can be calculated } \\
& B^{T} \bar{S}=\left[\begin{array}{llll}
1 & 4 & 6 & 4
\end{array}\right]
\end{aligned}
$$

This feedback gain choice is mainly motivated by the simplicity of the design procedure which is based on a sole parameter even in the case of multivariable systems [9]. Furthermore, the high bandwidth value of the process under consideration enables such a choice.

Of particular interest, the first term of the control law $\hat{u}^{*}(t)$ ensures both a perfect tracking performance capability as well as a feed-forward type action as regards the load current disturbances whose estimation are on-line delivered by the observer (8).

Using this expression (19) of $u(t),\|z(t)\|$ remains bounded and converges exponentially for high values of the design parameter $\lambda$.

\section{Proof}

The different steps of the proof have been proposed in [6] whenever the input non-linearity is exactly compensated which actually corresponds to the nominal but restrictive case $v_{e}(t)=v_{e}^{*}$. As a result, a few calculus lines have been omitted and the reader is invited to refer to [6] for more details.

Introducing the expressions (19), (20) and (21) of the control law in the control model (16), one obtains 


$$
\begin{aligned}
\dot{z}(t) & =A z(t)-\frac{v_{e}(t)}{v_{e}^{*}} k_{c} B B^{T} \bar{S} \Gamma_{\lambda} z(t) \\
& +B \frac{v_{e}(t)}{L}\left(\hat{u}^{*}(t)-u^{*}(t)\right)+\varphi(z(t))
\end{aligned}
$$

Let us define $\bar{z}(t)=\Gamma_{\lambda} z(t)$, then

$$
\begin{aligned}
\dot{\bar{z}}(t) & =\lambda A \bar{z}(t)-\frac{v_{e}(t)}{v_{e}^{*}} \lambda k_{c} B B^{T} \bar{S} \bar{z}(t) \\
& +\lambda B \frac{v_{e}(t)}{L} \tilde{u}^{*}(t)+\Gamma_{\lambda} \varphi(z(t))
\end{aligned}
$$

Set $V(t)=\bar{z}^{T}(t) \bar{S} \bar{z}(t)$ the Lyapunov candidate function. The first derivative of $V(t)$ can be written as follows

$$
\begin{aligned}
\dot{V}(t) & =2 \lambda \bar{z}^{T}(t) \bar{S} A \bar{z}(t)+2 \bar{z}^{T}(t) \bar{S} \Gamma_{\lambda} \varphi(z(t)) \\
& -2 \frac{v_{e}(t)}{v_{e}^{*}} \lambda k_{c} \bar{z}^{T}(t) \bar{S} B B^{T} \bar{S} \bar{z}(t) \\
& +2 \lambda \bar{z}^{T}(t) \bar{S} B \frac{v_{e}(t)}{L} \tilde{u}^{*}(t)
\end{aligned}
$$

Using the expression of $\bar{S} A$ deduced from the Lyapunov equation (22), one can easily obtain

$$
\begin{aligned}
\dot{V}(t) & =-\lambda V(t)+2 \bar{z}^{T}(t) \bar{S} \Gamma_{\lambda} \varphi(z(t)) \\
& +\lambda \bar{z}^{T}(t) \bar{S} B B^{T} \bar{S} \bar{z}(t)+2 \lambda \bar{z}^{T}(t) \bar{S} B \frac{v_{e}(t)}{L} \tilde{u}^{*}(t) \\
& -2 \lambda k_{c} \frac{v_{e}(t)}{v_{e}^{*}} \bar{z}^{T}(t) \bar{S} B B^{T} \bar{S} \bar{z}(t)
\end{aligned}
$$

Set the following change of variables $\xi(t)=\bar{S} \bar{z}(t)$. Then one has

$$
\begin{aligned}
\dot{V}(t) & =-\lambda V(t)+2 \bar{z}^{T}(t) \bar{S} \Gamma_{\lambda} \varphi(z(t)) \\
& +\lambda \xi^{T}(t) B B^{T} \xi(t)+2 \lambda \bar{z}^{T}(t) \bar{S} B \frac{v_{e}(t)}{L} \tilde{u}^{*}(t) \\
& -2 \lambda k_{c} \frac{v_{e}(t)}{v_{e}^{*}} \xi^{T}(t) B B^{T} \xi(t)
\end{aligned}
$$

which leads to

$$
\dot{V}(t)<-\lambda V(t)+2 \bar{z}^{T}(t) \bar{S} \Gamma_{\lambda} \varphi(z(t))+2 \lambda \bar{z}^{T}(t) \bar{S} B \frac{v_{e}(t)}{L} \tilde{u}^{*}(t)
$$

under the following condition

$$
\forall \xi(t) \quad k_{c}>\frac{1}{2} \frac{v_{e}^{*}}{v_{e \min }}
$$

Using (11), one obtains

$$
\begin{aligned}
\left\|\lambda B \frac{v_{e}(t)}{L} \tilde{u}^{*}(t)\right\| & \leq\left\|\lambda B \frac{v_{e \max }}{L} \tilde{u}^{*}(t)\right\| \\
& \leq \lambda L C\left(1+\frac{R_{L}}{L}\right)\left(\eta_{0}(\theta) e^{-\mu_{0}(\theta) t}\left\|\hat{x}_{0}(0)-x_{0}(0)\right\|+m \frac{\delta}{\theta}\right)
\end{aligned}
$$

With this inequality, we obtain an expression similar to [6] and we invite readers to see the end of the proof in this paper.

\section{SIMULATION RESULTS}

In this section, the integral high gain control law (19) is applied to a chopper buck converter supplied by a battery. Given the complexity of phenomena involved in electro- chemical battery, we will take an approximate dynamic model corresponding to an equivalent source with its internal impedance as described on figure 2 [7].

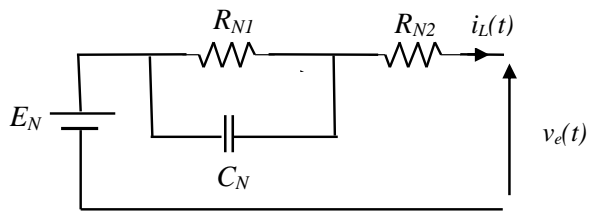

Fig. 2. Schematic of Battery.

The supplied voltage $v_{e}(t)$ by the battery depends on its charge level and supplied current with $v_{e}^{*}=v_{e \max }=E_{N}$ The variations of the voltage $v_{e}$ from the charge state of the battery are considered as ramp type disturbances.

In this application, the input voltage $E_{N}$ has been fixed to $14 \mathrm{~V}$ whereas the output voltage reference is $6 \mathrm{~V}$. Physical parameters of the battery and converter have been reported in Table 1.

TABLE I

MODEL PARAMETERS

\begin{tabular}{cccc}
\hline \hline Symbol & Quantity & Value & Unit \\
\hline$R_{N 1}$ & resistor & 0.2044 & $\Omega$ \\
$R_{N 2}$ & resistor & 0.3844 & $\Omega$ \\
$C_{N}$ & capacity & 0.3 & $\mathrm{~F}$ \\
$E_{N}{ }^{*}$ & input voltage & 14 & Volts \\
$L$ & inductance & 0.56 & $\mathrm{mH}$ \\
$C$ & capacity & 10 & $\mathrm{mF}$ \\
$R_{L}$ & resistor & 30 & $\mathrm{~m} \Omega$ \\
$u_{\max }$ & used in control & 0.98 & - \\
$u_{\min }$ & used in control & 0.02 & - \\
$v_{c}{ }^{*}$ & Operating voltage & 6 & Volts
\end{tabular}

The source voltage profile $E_{N}(t)$ including a ramp disturbance from $E_{N}(t)=14 \mathrm{~V}$ to $E_{N}(t)=12.5 \mathrm{~V}$ from time $\mathrm{t}=10 \mathrm{~s}$ up to time $\mathrm{t}=50 \mathrm{~s}$ is presented on figure 3 . The figure 4 shows the load resistor $R(t)$ profile. This profile imposes the following load current one $i(t)=v_{c}^{*} / R(t)$. It consists of two steps and two ramps in opposite sign filtered by a second order transfer function (dumping factor $=1$ and $\left.\omega_{\mathrm{n}}=60 \mathrm{rd} / \mathrm{s}\right)$. The supply voltage disturbance occurs when the after load current one has been effectively rejected. A steplike disturbance with magnitude $0.5 \mathrm{~V}$ is applied to the output voltage at $t=50$ s. Finally, the output voltage values have been corrupted by a measurement noise with zero mean value and a standard deviation equal to $0.00025 \mathrm{~V}$.

Several simulations have been carried out in order to obtain the optimal control design parameters as regards the input and output performances. The following values have been selected:

$$
\lambda=50, \theta=50, \mathrm{k}_{\mathrm{c}}=5
$$

The input voltage $v_{e}(t)$ is presented on figure 5 . The voltage firstly quickly drops because of the impedance of the battery. On the other hand, the observed variations beginning at time $\mathrm{t}=35 \mathrm{~s}$ are due to the ramp like disturbance applied to $E_{N}(t)$. 
The output voltage $v_{c}(t)$ and the estimated output voltage performance are shown in figures 6 and 7. The results are presented on two separated figures to see with more accuracy the effect of the different disturbances. Figure 6 shows the good performances of the control law. In fact, the effects of the load current and the supply voltage perturbations are below the measurement noise magnitude. Of particular interest, the double integral action obviously ensures the static error to be null. In Figure 7, we see that the control law rejects efficiently the output disturbance voltage with the dynamics of the observer.

The other simulation results are shown in figures 8 to 11 . In particular, the control variable is presented on figure 11. The input performances are relatively well filtered whereas the output voltage values have been corrupted by noise measurements. Finally, the coil current (figure 9) profile follows the load current one (figure 8) as we stated it for the control law synthesis.

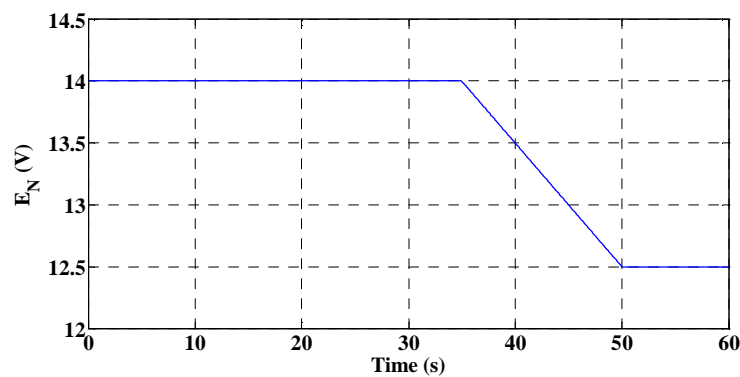

Fig. 3. : Supply voltage $E_{N}(t)$ simulation profile

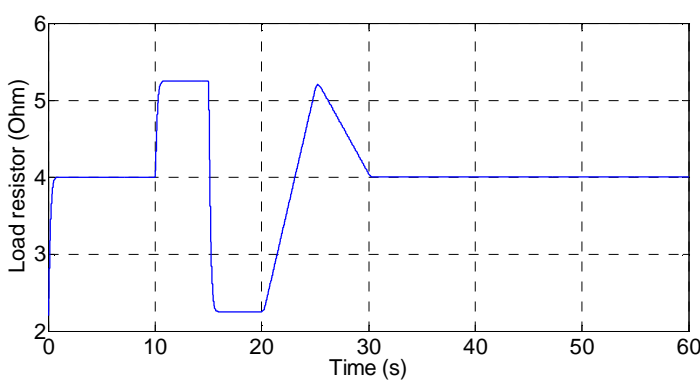

Fig. 4. Load resistor simulation profile.

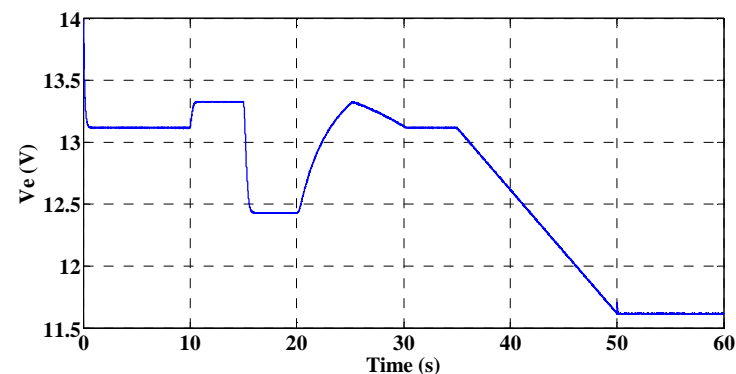

Fig. 5. Input Voltage $v_{e}(t)$.
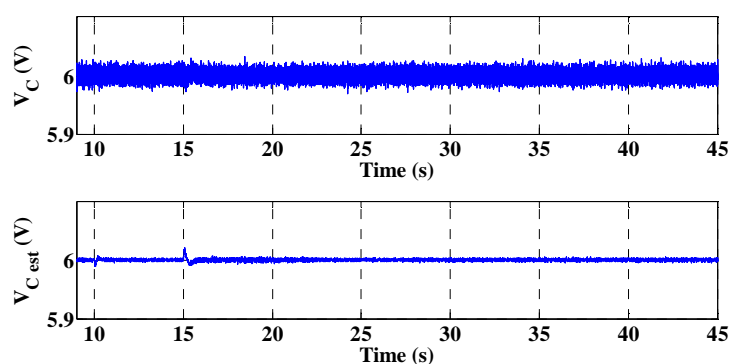

Fig. 6. Output Voltage $v_{c}(t)$ and $\hat{v}_{c}(t)$.
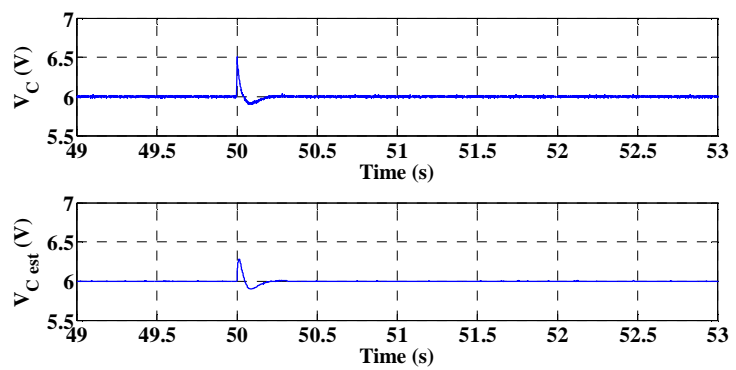

Fig. 7. Output Voltage $v_{c}(t)$ and $\hat{v}_{c}(t)$.

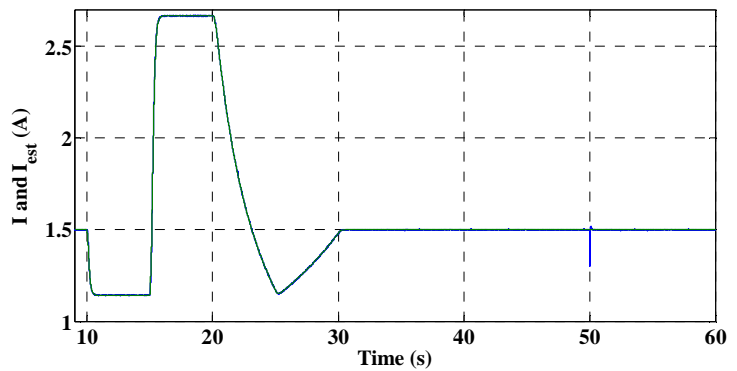

Fig. 8. Load current $i(t)$ and $\hat{i}(t)$.

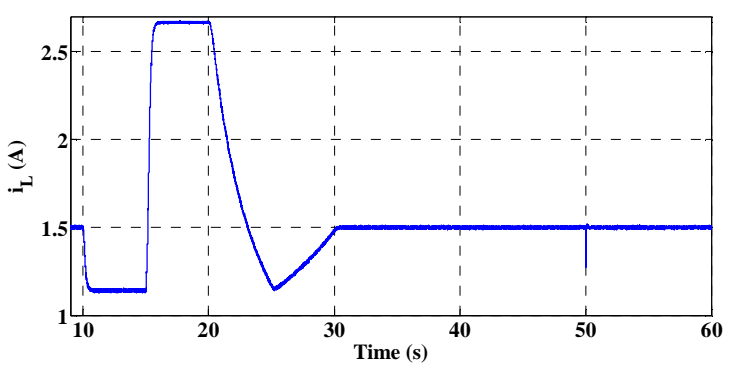

Fig. 9. Coil current $i_{L}(t)$.

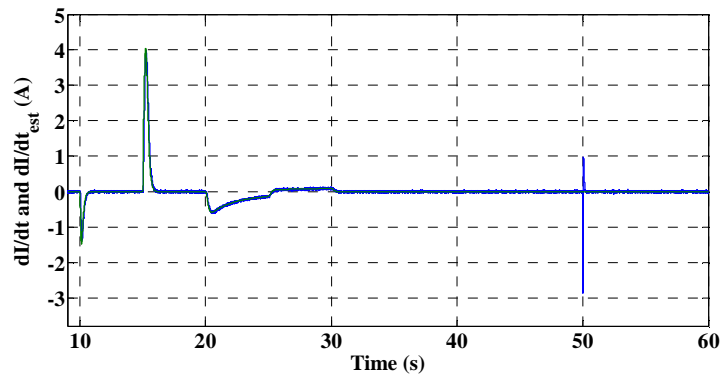

Fig. 10. Derivative load current $d i(t) / d t$ and its estimation. 


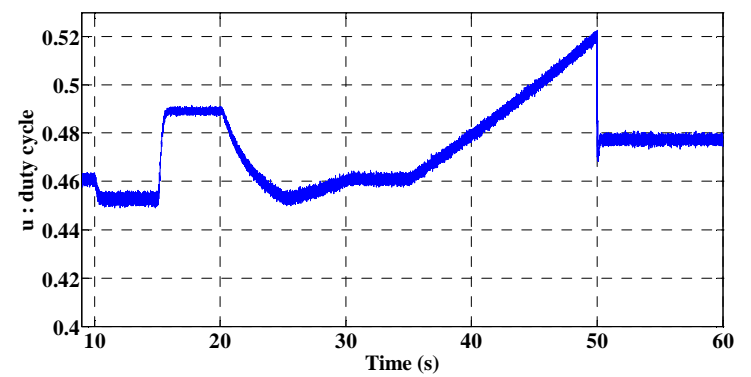

Fig. 11. Duty cycle $u(t)$.

\section{CONCLUSION}

In this paper, we have presented a non linear control law for buck DC/DC static converter. The high gain state feedback controller has been synthesized using the nonlinear model. The main interest of this approach comes from the fact that globally exponentially stability of the control scheme has been proved for the considered class of model. Of great interest the load current disturbances are exactly rejected and the control scheme deals with the drift of the voltage supply without any additional sensor. Numerical simulations have been carried out and the obtained results confirm the good performances of the proposed control scheme. Experimental evaluation of the control algorithm is actually under investigations.

\section{REFERENCES}

[1] R.D. Middlebrook and S. Cuk, "A general unified approach to modeling switching-converter power stages", in Proc. IEEE Power Electron. Specialists Conf. (PESC) 1976, pp 18-34.

[2] H. Sira-Ramirez, R. Perez-Moreno, R. Ortega, and M. Garcia-Esteban, 'Passivity-based controllers for the stabilisation of DC-to-DC power converters', Automatica, 1997.

[3] H. Maker, H. Gualous and R. Outbib, 'Sliding Mode Control with Integral of Boost Converter by Microcontrollerr for a class of nonlinear systems', in Proc Int Conf on Control Applications, October 2006, pp. 3134-3138

[4] H. El Fadil, F. Giri, M. Haloua and H. Ouadi, 'Non linear and adaptive control of buck power converter', in Proc. IEEE Decision and Control, Hawaï, Dec 2003, pp 4475-4480.

[5] M. Fliess and H. Sira-Ramirez, 'Regulation of nonminimum phase outputs: a flatness based approach', Springer-Verlag, NY, 1998.

[6] A. Bédoui, M. Farza, M. M'Saad and M. Ksouri, "Robust nonlinear controllers for bioprocesses" in Proc. $17^{\text {th }}$ World Congress IFAC, Seoul, Korea, July 6-11, 2008.

[7] M. B. Camara, H. Gualous, F, Gustin and A. Berthon, "Design and new control of DC/DC Converters to share energy between supercapacitors and batteries in hybrid vehicles", IEEE Trans on vehicular technology, Vol 57, N5, sept 2005.

[8] M. Farza, M. M'Saad and L. Rossignol, "Observer design for a class of MIMO nonlinear systems", Automatica, 40(1), 135-144, 2004.

[9] M. Farza, M. M'Saad, O. Gehan, E. Pigeon and S. Hajji, "State feedback controller for a class of MIMO non triangular systems" accepted at $18^{\text {th }}$ World Congress IFAC, Milan, Italy, August 28 September 02, 2011.

[10] M. Farza, K. Busawon, and H. Hammouri, "Simple Nonlinear observers for on-line estimation of kinetic rates in bioreactors", Automatica, 34 (3), pp 301-318, 1998. 